\title{
Pertinencia territorial de las críticas al culturalismo
}

Mariflor Aguilar Rivero Universidad Nacional Autónoma de México

Resumen

La diversidad cultural ha ido ganando visibilidad, y con ello los movimientos sociales que de varias formas buscan hacerse escuchar. Al mismo tiempo se fue configurando el área de trabajo e investigación sobre los estudios culturales que buscaron abrir brecha para el reconocimiento de la identidad de las culturas. Sin embargo, en la línea contraria se desarrollan líneas de análisis que cuestionan los mismos estudios culturales. En este trabajo me voy a referir a algunos de los argumentos críticos al culturalismo para después tratar de pensar desde ellos la eficacia justificatoria de procesos de acumulación originaria que todavía azotan al país.

Palabras clave: identidad, diferencia, culturalismo, ciudad rural, racismo.

Abstract

Cultural diversity has been increasing visibility and so are social movements searching to be heard. At the same time cultural studies have conformed a special area for studies and research that have been contributing to the recognition of culture identities. Nevertheless, in the opposite way, there have been developing some lines of argument which are calling into question the very cultural studies. In this paper I'll expose some of these critical lines of thought against culturalism in 
order to throw some light from them to part of the land plundering that is being practiced in Mexico all around.

Keywords: identity, difference, culturalism, rural city, racial discrimination

D or diferentes circunstancias, la diversidad cultural fue ganan1 do visibilidad, y con ella los movimientos sociales y culturales que de varias maneras intentaban hacerse escuchar. Junto con éstos también se fue configurando el área de trabajo e investigación sobre los estudios culturales que buscaron abrir brecha para el reconocimiento de culturas y valores múltiples y para la reivindicación de las luchas por el reconocimiento de su identidad. Sin embargo, en la línea contraria se desarrollaron líneas de análisis que cuestionan los mismos estudios culturales. En este trabajo me voy a referir a algunos de los argumentos críticos al culturalismo, para después tratar de pensar desde ellos la eficacia justificatoria de procesos de acumulación originaria que todavía azotan al país.

De las críticas al culturalismo me referiré en particular a una, cuyo portavoz es sobre todo S. Zizek, tiene cuatro modos. El primer modo considera que el multiculturalismo es la contraparte ideológica de la globalización, en el sentido de que los Estados globales dan origen a nuevas identidades y promueven mundos diversos mediados por la universalidad del mercado. Asume que mientras nuestras batallas giran sobre los derechos de las minorías o los diferentes estilos de vida, "el capitalismo continúa su marcha triunfal" (Zizek, 1998: 171).

Lo anterior se articula con el segundo modo crítico zizekiano, que aunque se enfila contra los descentramientos y las identidades nómadas de Deleuze y Foucault, forma parte de la misma problemática. Desde ese lugar considera el esloveno que la diversidad de culturas y la multiplicidad de identidades implicadas en la tesis del descentramiento del poder ganaron en cantidad y perdieron en 
cualidad, ya que lo que proliferaron fueron moléculas todas iguales entre sí reducidas a micropoderes como fuerzas de subjetivación. Si bien, entiende que los avances teóricos de Foucault no se pueden ni se deben soslayar, asimismo comprende que lo que ahora se requiere es que esas instancias de micropoder se vean acompañadas también de economía; tal vez podría hablarse de "instancias de microeconomía" o "microplusvalía”. Cuando Zizek observa que "más que nunca, el Capital es el 'universal concreto' de nuestra época histórica" (Zizek, 2006: 211), consideramos que también significa que el capital —o la plusvalía_ “ "penetra los cuerpos”.

La tercera versión de esta crítica es igual a la anterior y está implicada en ella, sólo que en lugar de decir que a la multitud de identidades del multiculturalismo le hacen falta "instancias de microeconomía", lo que ahora se echa en falta son las clases sociales; la multiplicidad, en este caso, dice Zizek, "se afirma frente al espectro del anticuado 'esencialismo y reduccionismo de clase", y al hacerlo desaparece la "clase" (Zizek, 2006: 224). Otra manera de decir lo mismo es señalando que el discurso culturalista sustituye al discurso político (Balibar, 1991: 58).

Por último, el cuarto modo de la crítica zizekiana es pensada en los términos freudianos de la estructura de Eros y Tánatos del Malestar en la cultura, que dice que "ante cada afirmación de Eros, Tánatos se reafirma con una venganza”. Según esto, el discurso multiculturalista es una pseudoafirmación de Eros que toma la forma de la tolerancia del "hedonismo estetizante", de los "estilos de vida", y éste es un modo complementario del discurso del poder que pretende moverse en un universo postideológico de negociaciones racionales entre las diversidades (Zizek, 2006: 156-7).

Quizá estos cuatro modos pueden resumirse en una tesis sobre la causalidad propuesta por Zizek: en los sistemas complejos, como es la sociedad, hay que distinguir entre explicaciones monocausales simplistas y la pluricausalidad homogénea. Ambas deben 
rechazarse. No debe subsumirse la explicación de las prácticas y procesos locales a una sola causalidad, pero tampoco puede pensarse que todas las causalidades juegan el mismo papel de distribución (Zizek, 2007: 169-70).

Lo interesante de este tipo de críticas es que representan una vuelta de tuerca a la "ontología política". Como se dijo antes, se reconoce que es un paso adelante respecto de la microfísica del poder y de las fuerzas; dice el filósofo psicoanalista:

Sin duda, hay que reconocer el importante impacto liberador de la politización postmoderna en ámbitos hasta entonces considerados apolíticos [...] No se trata, por tanto, de minusvalorar estos desarrollos para anteponerles alguna nueva versión de esencialismo económico $[\ldots]$.

Dicho de otra forma, y pasando al segundo punto que quiero tratar, se trata de poner límite a la filosofía de la diferencia ahí donde ésta gire de ser vehículo de reivindicaciones a ser obstáculo para ver exclusiones. $Y$ es esto lo que puede ocurrir con las diferencias multiculturales, a saber, que el discurso de la diferencia tienda a identificarse con lo llamado el "nuevo racismo", que toma la forma de "racismo diferencialista", o sea, un discurso que traslape la diferencia con la exclusión.

Pero, ¿cómo es esto?, ¿cómo ocurre que el discurso emancipador de las diferencias pueda coincidir con un discurso excluyente?, ¿cómo puede el discurso diferencialista contribuir a la exclusión? Puede decirse que para que esto ocurra opera como un puente efectivo la noción de "alteridad". Ser "lo otro", lo diferente, ha significado cosas distintas, como se sabe; ha tenido sentido positivo y negativo; ha significado ser lo extraño, lo "bárbaro", lo intrusivo y, por ello, sospechoso o desconfiable; y ha sido también equivalente a tener una naturaleza distintiva y diferencial, a tener una singularidad que constituya una identidad que se busca 
defender cuando es amenazada. Por esto las cuestiones de la interculturalidad, los problemas de género y las políticas de minorías han concedido al pensamiento de la diferencia y la alteridad un lugar central. Pero si bien es indispensable pensar las formas específicas de exclusión y de marginación, y no olvidar que las diferencias tienen su propia historia, se requiere también pensar que la carga negativa se desliza para transformar la diferencia en $l o$ otro que debe ser tolerado, en un sentido de tolerancia que va de la distancia y la amenidad (Richard, 1977: 118-9) a lo que no es como yo, y que está bien, pero siempre y "cuando se trate solamente de una cuestión de alimento, cultura y danzas" (Zizek, 2001). Es en ese deslizamiento que el discurso de la diferencia sufre - o puede sufrir - la metamorfosis hacia el discurso racista.

El tercer paso a dar ahora es plantear la segunda metamorfosis que va de un racismo convencional a un racismo sin razas que toma cuerpo en "población excedente", que son los desempleados y cada vez más también los migrantes (Balibar, 1991: 37). En virtud del proceso de disolución de algunos signos sociales estructurales en el seno de las diferencias homogéneas, la diferencia del racismo basado en la raza pasa por el miedo al extranjero (Zizek, 2010), se detiene en quienes son identificados como exteriores a la nación o a la cultura (Balibar, 2005: 75), y se estaciona en la exterioridad de los que en general son población laboral, personas desplazadas y desterritorializadas (no en el sentido deleuziano, sino literal).

Desde aquí continúa el deslizamiento semántico sobre los grupos sociales, y la exterioridad de los migrantes se superpone con sectores excluidos, o como lo formula Etienne Balibar, con sectores que forman una "unidad identitaria" que reúne a los pobres ya siempre excluidos con los "nuevos pobres", que son los extranjeros (o bárbaros). Así, desde este ángulo, la perspectiva territorial comienza a adquirir relevancia para cuestionar al culturalismo. Esta dimensión se vuelve más importante en el momento 
actual, pues la organización del mercado mundial ha generado gran cantidad de fenómenos sociales vinculados con el territorio y la movilidad de grupos humanos: desplazamientos, migraciones, reubicaciones, recolocaciones, etcétera, razón por la cual se reconoce ahora, y es mayor el significado cultural y social del territorio, acerca del cual pueden verse tres dimensiones: 1) un espacio de inscripción y distribución de instituciones y prácticas culturales, 2) un lugar como cobijo y/o sobrevivencia y 3) un objeto de apego afectivo como símbolo de pertenencia socioterritorial (Giménez, 1996: 8). Estas dimensiones de lo territorial propician que los procesos migratorios afecten "lo más cotidiano: la alimentación, casa, vestido, concepciones del mundo, y también, lo colectivo y público" (Duarte y Coello, 2007). Debido al carácter esencial y a su trascendencia, la repercusión del territorio es integral, ya que forma y transforma la inseparable triada subjetividad-entorno-cultura.

Es por ello que los problemas vinculados con él inciden frecuentemente en el sentido de "pertenencia" de los sujetos, en su autoimagen y en la manera como se identifican entre sí. Cuando los desplazamientos son forzados llegan a fracturar de manera irreparable las formas básicas de relación dentro de la sociedad, y de ésta con la naturaleza; el tejido social se desarticula, y se vulnera la cohesión de los grupos, lesionando a las comunidades y las relaciones construidas en tiempos largos que se tornan difíciles de recomponer.

Este modelo opera tanto en el caso de los mexicanos que masivamente han salido hacia el norte, como en el caso de las migraciones internas forzadas, bien sea por desastres "naturales", por reestructuración territorial, la que se impone como designio desde fuera sin considerar el interés de los grupos desplazados:

En Guatemala y Chiapas, los efectos de la pérdida de las tierras de las comunidades indígenas, bien sea por despojo o por desastres 
naturales, ha afectado la vida de las comunidades por la tendencia a la sobreexplotación de los recursos naturales y el deterioro de sus sistemas tradicionales de producción (Duarte y Coello, 2007: internet).

Si bien es cierto que según estudios diversos no toda migración deja huella traumática, sí puede inferirse que si el territorio juega un rol de importancia crucial en la conformación de la subjetividad, y si los individuos se ven obligados a desplazarse dejando atrás puntos de referencia que los constituyen en su singularidad y en su generalidad, entonces debe afirmarse que toda migración forzada, o no deseada, con seguridad dejará una huella traumática, y lamentablemente esto incluye a las migraciones internas $y$, por tanto, a la estructura nacional.

Digo esto porque, como se sabe, son piezas importantes de la estructura económica nacional dos tipos de migraciones: una, la más conocida, la de los ocho millones de mexicanos que salieron y se quedaron en Estados Unidos; otra, son las migraciones internas - o reubicaciones - que son parte del Plan Nacional de Desarrollo; es también una de las políticas clave recomendada en la Estrategia para el Desarrollo de los Estados del Sur (EDES) del Banco Mundial (Wilson, 2008), y es parte medular del Plan del Estado Chiapas Solidario.

En su primer informe de gobierno, el gobernador de Chiapas se refirió a estos movimientos estratégicos del modo siguiente:

El Gobierno de Chiapas busca, a través de estas acciones, conjuntar esfuerzos con diversas instancias e impulsar el magno proyecto de Ciudades Rurales, único en su tipo en el país y ejemplo para otras entidades federativas, que así como Chiapas, enfrentan a la marginación, resultado de la dispersión poblacional (Sabines, 2007, internet). 
No es el lugar ni el momento para hablar con detalle de estas ciudades rurales, una de las políticas públicas más promovidas e impulsadas por los gobiernos federal y estatales en los últimos tres años, pues en diferentes trabajos anteriores ya hemos dando cuenta de ello. Más bien, lo que aquí queremos destacar es solamente un aspecto más para completar el argumento. Este aspecto es el relacionado con la dimensión "culturalista", con el que se quiere apuntalar el proyecto. Hay que decir, ciertamente, que no es la dimensión cultural la que juega el papel central del "blindaje mediático y discursivo" que afianza esta política pública, sino es la económica y el discurso de combate a la pobreza. No obstante, el discurso referido a las ciudades rurales enfatiza hasta el sinsentido a la cultura como uno de los rubros que se busca mejorar con ese programa.

En el foro donde se presentó ante la academia el proyecto de las ciudades rurales se dijo que uno de sus objetivos es favorecer el arraigo al entorno y respetar la cultura de las comunidades. De ello sorprenden tres cosas: primero, que para favorecer el arraigo al entorno antes se tenga que desarraigar a la gente; segundo, que se hable de respetar la cultura de las comunidades como si la cultura no formara parte, o más aún, no fuera ella misma el modo cultural de vida del que se desarraiga a la gente; y tercero, que el entorno de estas ciudades rurales, al menos de la única que lleva funcionando más de un año, que es la de Nuevo Juan de Grijalva, en Chiapas, en el municipio de Ostuacan, tenga como mayor instancia la presencia de Fundación Azteca, a través de la cual el Estado se encargará de "promover" y "fortalecer" la cultura. Esta Fundación implantó un enorme y llamativo monumento dedicado al Teletón en la entrada del centro de salud (cortesía Teletón), y cuenta ya con una torre central llamada Torre Azteca, que se trata de una "torre inteligente" porque en ella se dispone de un salón con computadoras para el uso de la población; es también donde los funcio- 
narios reciben a los observadores nacionales e internacionales. A Fundación Azteca se le considera, junto a Telmex, una "sociedad civil organizada" que "acompañará” y evaluará el proceso. Se tiene planeado que esta empresa instale una sucursal del Banco Azteca, y que eche a andar el Programa Empresario Azteca para la promoción y fortalecimiento de las PYME (Pequeña y Mediana Empresa).

Si se piensa que la Fundación Azteca es la cara proselitista de uno de los monopolios televisivos de los que depende una parte importante de la educación del pueblo mexicano, y que junto con Televisa son empresas especializadas "en el sutil arte de engañar con la verdad" (Fazio, 2010); si, por otra parte, se toma conciencia de que esa "educación" va en la dirección del humo del festejo frenético de lo "logrado", no es difícil entonces percatarse de la concepción de "cultura" supuesta en el programa Ciudades Rurales, como un producto para el consumo, estandarizado a nivel nacional y enajenante, asimismo como parte estructural del blindaje mediático y discursivo que legitima políticas públicas que aparentemente, y también declarado por los mismos promotores, tienen como objetivo la inserción de los sectores rurales en la economía de mercado. Frente a estas prácticas sociales es ahora importante tomar con pinzas la noción de "cultura" cada vez que en su nombre se tomen decisiones que impactan a grupos poblacionales. De igual forma, se vuelve una exigencia entender que la cultura son también modos de vida que, antes de ser destruidos en nombre del progreso y la modernización, habría que observar las enseñanzas que siguen ofreciendo a los modernos.

\section{Bibliografía}

Aguilar, Mariflor, 2009, "Midiendo el progreso de las sociedades. Entre la libertad y la sobrevivencia”, en Midiendo el progreso de las socieda- 
des. Reflexiones desde México (ed. de Mariano Rojas), Foro Consultivo Científico Tecnológico, México, 2008 (Measuring the progress of societies. Reflections from Mexico).

Balibar, Etienne, 1991, “¿Existe un neorracismo?”, en Etienne Balibar e Immanuel Wallerstein, Raza, nación y clase, Iepala, Textos: 16, Madrid, $360 \mathrm{pp}$.

_- 2005, Violencias, identidades, civilidad (trad. de Luciano Padilla), Gedisa, Barcelona.

Duarte, Rolando, y Teresa Coello, 2007, "La decisión de marcharse: los pueblos indígenas migrantes de Guatemala y Chiapas", Consejería en Proyectos. http://www.iidh.ed.cr/BibliotecaWeb/Varios/Documentos/BD_1961302194/Migracionesindgusa.doc (consulta en 2008).

Fazio, Carlos, 2010, “De misterios y apariciones", en La poca madre de los poderosos, s.e., s.l.e.

http://pocamadrenews.wordpress.com/2010/12/27/de-misterios-y-apariciones-por-carlos-fazio/ (consulta el 31 de marzo de 2011).

Giménez, Gilberto, 1996, "Territorio, cultura, identidades. La región sociocultural”, en Estudios sobre las culturas contemporáneas, vol. II, núm. 004, Universidad de Colima, México, diciembre, pp. 9-30.

Nelly Richard, 1998, "Intersectando Latinoamérica con el latinoamericanismo: discurso académico y crítica cultural", en Teorías sin disciplina (latinoamericanismo, poscolonialidad y globalización en debate) (ed. de Santiago Castro-Gómez y Eduardo Mendieta), Miguel Ángel Porrúa, México, pp. 118-9.

Sabines Guerrero, Juan, 2007, Primer Informe de Gobierno, 10 de diciembre, Chiapas.

Slavoj, Žižek, 1998, "Multiculturalismo o la lógica cultural del capitalismo multinacional”, en F. Jameson y Slavoj Zizek, Estudios culturales. Reflexiones sobre el multiculturalismo, Paidós, Buenos Aires. 
__ 2001, "La medida del verdadero amor es: puedes insultar al otro", entrevista a S. Zizek por Sabine Reul y Thomas Deichmann, en Palabrerio, núm. 1. http://www.palalbedrio.com.ar/externos/zizek2.htm.

—, 2006 , Órganos sin cuerpos. Sobre Deleuze y consecuencias, Pre-textos, Valencia.

—_, 2007, En defensa de la intolerancia, Sequitur, Madrid.

—_, 2010, "Barbarie con rostro humano", en El Pais, Madrid, 23 de marzo. http:/www.elpais.com/articulo/opinion/Barbarie/rostro/humano/elpepiopi/20101023elpepiopi_10/Tes.

Japhy Wilson, 2008, "La nueva fase del plan Puebla-Panamá en Chiapas" (segunda de tres partes), en Boletines de CIEPAC Chiapas al Dia, Chiapas, México, núm. 561, 27 de mayo. 